\title{
Influência do trabalho de docência no bem-estar individual, qualidade de vida, e (in) atividade física de professoras do ensino fundamental
}

\author{
Influence of teaching work on individual well-being, quality of life, and (in) physical activity of \\ elementary teachers \\ Influencia del trabajo docente en el bienestar individual, la calidad de vida y la (in) actividad física \\ de los profesores de primaria
}

\author{
Jaqueline Carvalho Cirilo \\ ORCID: https://orcid.org/0000-0001-9286-6714 \\ Secretaria Municipal de Educação, Brasil \\ E-mail: jaque.ed.fisica@hotmail.com \\ David Michel de Oliveira \\ ORCID: https://orcid.org/0000-0003-0658-7693 \\ Universidade Federal de Jataí, Brasil \\ E-mail: profdoliveira@ufj.edu.br \\ Eduardo Vignoto Fernandes \\ ORCID: https://orcid.org/0000-0003-0876-2491 \\ Universidade Federal de Jataí, Brasil \\ E-mail: eduardovignoto@ufj.edu.br \\ Anderson Geremias Macedo \\ ORCID: https://orcid.org/0000-0001-7076-0900 \\ Universidade Estadual Paulista "Júlio de Mesquita Filho", Brasil \\ E-mail: andersongmacedo@yahoo.com.br \\ Daniel dos Santos \\ ORCID: https://orcid.org/0000-0002-5565-2351 \\ Universidade de Franca, Brasil \\ E-mail: daniel.santos@unifran.edu.br
}

\begin{abstract}
Resumo
Introdução: O trabalho docente é imprescindível para sociedade, mas de alta exigência e baixa valorização o que pode interferir na saúde de docentes. Objetivo: avaliar a influência do trabalho docente no estilo, qualidade de vida (QV), e nível de atividade física (AF) de professores. Métodos: Estudo transversal avaliou docentes do ensino fundamental de escolas públicas. Foram utilizados instrumentos autopreenchidos pelos participantes sobre o perfil profissional, massa corporal, estatura autorreferidos, e calculado o índice de massa corpórea (IMC), para o perfil do estilo de vida individual (PEVI) utilizou-se Pentáculo do Bem-estar, a QV foi avaliada pelo Questionário de Qualidade de Vida SF-36, e o nível de AF pelo International Physical Activity Questionnaire - IPAQ. Resultados: Participaram 93 docentes do sexo feminino com idade média de $(43,5 \pm 7,8)$ anos, IMC $(26,9 \pm 4,8)$, com carga horária semanal de $(40,4 \pm 13,4)$ horas e tempo de serviço de $(16,5 \pm 7,9)$ anos. Sobre PEVI, a nutrição $(1,57 \pm 0,8)$, AF $(0,94 \pm 0,9)$, e controle do estresse $(1,68 \pm 0,9$. Em relação a QV estão próximas aos valores mínimos de referência. 73,2\% das docentes foram classificadas pelo IPAQ como irregularmente ativas ou sedentárias. Não foram encontrados correlação entre as variáveis. Foi observado associação $\mathrm{p}(0,01)$ entre AF, tempo de docência e PEVI. Conclusão: Foi possível concluir que embora as professoras tenham sido consideradas com boa QV, a maioria foi classificada com sobrepeso, estilo de vida abaixo do nível saudável e insuficientemente ativas. Estratégias institucionais devem ser implantadas para prevenção do adoecimento docente.
\end{abstract}

Palavras-chave: Professores escolares; Qualidade de vida; Comportamento sedentário; Atividade motora.

\begin{abstract}
Introduction: The teaching work is essential for society, but of high demand and low valuation, which can interfere with the health of teachers. Objective: to evaluate the influence of teaching work on the style, quality of life (QL), and level of physical activity (PA) of teachers. Methods: Cross-sectional study evaluated elementary school teachers from public schools. Self-reported instruments were used by the participants on the professional profile, body mass, height, and the body mass index (BMI) was calculated. was assessed by the Quality of Life Questionnaire - SF-36, and the PA level by the International Physical Activity Questionnaire - IPAQ. Results: 93 female teachers participated, with a mean age of (43.5 \pm 7.8$)$ years, BMI (26.9 \pm 4.8$)$, with a weekly workload of $(40.4 \pm 13.4)$ hours and service time of
\end{abstract}


$(16.5 \pm 7.9)$ years. Regarding PEVI, nutrition ( $1.57 \pm 0.8)$, PA (0.94 \pm 0.9$)$, and stress control (1.68 \pm 0.9 . Regarding QoL, they are close to the minimum reference values $73.2 \%$ of the teachers were classified by the IPAQ as irregularly active or sedentary. No correlation was found between the variables. There was an association $\mathrm{p}(0.01)$ between PA, teaching time and PEVI. Conclusion: It was possible to conclude that although teachers were considered to have a good QoL, most were classified as overweight, a lifestyle below a healthy level and insufficiently active Institutional strategies should be implemented to prevent teachers from falling ill.

Keywords: School teachers; Quality of life; Sedentary Behavior; Motor activity.

\section{Resumen}

Introducción: La labor docente es fundamental para la sociedad, pero de alta demanda y baja valoración, lo que puede interferir en la salud de los docentes. Objetivo: evaluar la influencia del trabajo docente en el estilo, la calidad de vida (CV) y el nivel de actividad física (AF) de los docentes. Métodos: Estudio transversal que evaluó a profesores de primaria de escuelas públicas. Los participantes utilizaron instrumentos autoinformados sobre perfil profesional, masa corporal, estatura y se calculó el índice de masa corporal (IMC). Se evaluó mediante el Cuestionario de Calidad de Vida - SF-36, y el nivel de AF por el International Cuestionario de actividad física - IPAQ. Resultados: participaron 93 profesoras, con una edad media de $(43,5 \pm 7,8)$ años, IMC $(26,9 \pm 4,8)$, con una carga de trabajo semanal de $(40,4$ $\pm 13,4)$ horas y tiempo de servicio de $(16,5 \pm 7,9)$ años. En cuanto a PEVI, nutrición $(1,57 \pm 0,8)$, AF $(0,94 \pm 0,9)$ y control del estrés $(1,68 \pm 0,9)$. En cuanto a la $\mathrm{CV}$, se acercan a los valores mínimos de referencia 73,2\% de los docentes fueron clasificados por el IPAQ como irregularmente activos o sedentario. No se encontró correlación entre las variables. Hubo asociación p (0.01) entre AF, tiempo lectivo y PEVI. Conclusión: Se pudo concluir que si bien se consideró que los docentes tenían una buena $\mathrm{CV}$, la mayoría fueron clasificados como con sobrepeso, un estilo de vida por debajo de un nivel saludable e insuficientemente activo Se deben implementar estrategias institucionales para evitar que los docentes enfermen.

Palabras clave: Maestros; Calidad de vida; Conducta Sedentaria; Actividad del motor.

\section{Introdução}

Vários fatores relacionados ao mercado de trabalho docente têm colaborado para o desenvolvimento de estresse, depressão, baixa qualidade de vida dos professores do ensino público (Ribeiro et al. 2018). Esses profissionais apresentam grande quantidade de horas de trabalho e baixa média salarial, embora maior que maior que a média da população brasileira, de modo geral os professores exercem docência em vários locais ou utilizam outros meios para gerarem renda extra (Delcor, 2004; Reis, 2006). Outro fator importante é a condição de trabalho, como falta de recursos, materiais, equipamentos adequados e violência escolar causam dificuldades para estes profissionais desempenharem sua produtividade (Delcor, 2004; Reis, 2006). Por último o fator social devido a desvalorização da sociedade em relação aos professores e incentivo governamental (Silva et al. 2018).

Além disso, estas mudanças físicas e mentais podem afetar diretamente o estilo e qualidade de vida desta classe profissional (Oliveira, 2015, Coutinho et al., 2013). Fatores comportamentais e ambientais juntamente com a inatividade física podem desencadear a obesidade e já tem sido mostrado a prevalência de sobrepesos e obesos de professores municipais (Oliveira et al. 2011; Ribeiro et al. 2018). Conforme observado o ambiente e fatores ligados as atividades laborais dos professores do ensino público fundamental pode desencadear o comportamento sedentário e influenciar o estilo de vida e a qualidade de vida destes profissionais.

Desta forma, o objetivo deste trabalho foi investigar as características das professoras do ensino fundamental I do município de Barretos-SP no que tange ao seu estilo de vida, a qualidade de vida e o nível de atividade física

\section{Materiais e Métodos}

Esta pesquisa de caráter descritivo transversal e quantitativo segundo Pereira et al. (2018) foi realizada em 19 escolas municipais do Ensino Fundamental I do $1^{\circ}$ ao $5^{\circ}$ ano do município de Barretos, localizada na mesorregião de Ribeirão Preto da região Centro-Oeste do estado de São Paulo, Brasil, com nº 122.098 habitantes (IBGE, 2019), e com escolas públicas municipais de ensino fundamental (Secretaria Municipal da Educação, 2021). 
Foram convidados para participar do estudo 242 docentes de ambos os sexos, de todas as escolas da rede pública municipal de Barretos-SP. Utilizou-se os seguintes critérios de inclusão: possuir carga horária igual ou superior a 20 horas de trabalho semanal; estar atuando há no mínimo três anos como docente; possuir idade inferior ou igual a 60 anos; serem do sexo feminino, pois apenas 2 docentes do sexo masculino foram identificados inicialmente, segundo os dados fornecidos pela Secretaria Municipal de Educação. Critérios de exclusão. Destes, apenas 93 docentes (38,42\%) atenderem aos critérios de inclusão, aceitaram a participar da pesquisa e assim assinaram o termo de consentimento livre e esclarecido (TCLE) após serem esclarecidos sobre os procedimentos bioéticos.

\section{Procedimentos}

A coleta foi realizada no período de março a novembro de 2017. Primeiramente a pesquisa foi divulgada por meio de e-mail enviado para a direção das escolas e posteriormente foi realizada reunião com as coordenadoras para esclarecimentos da pesquisa. Os instrumentos foram entregues aos participantes para preenchimento durante o momento destinado a reuniões de trabalho pedagógico coletivo.

\section{Instrumentos}

Para avaliar o perfil profissional das participantes foi utilizado um questionário semiestruturado contendo as seguintes variáveis descritivas: identificação, idade, sexo, local de trabalho, carga horária semanal e tempo no exercício da função como docente. Foram autorreferidos a massa corporal e estatura, e depois calculado o IMC em kg/m² pelos pesquisadores utilizando os valores de referência estabelecido pela WHO (1995).

Para análise do perfil do estilo de vida individual (PEVI) foi utilizado o "Pentáculo do Bem-Estar", o instrumento é composto por 15 itens, que avaliam cinco aspectos no formato de mapa individual, representadas pela figura de um pentáculo que ilustra cinco componentes do estilo de vida: nutrição, $\mathrm{AF}$, comportamento preventivo, relacionamento social e controle do estresse. Cada componente tem 3 perguntas com os seguintes valores e classificações: 0 a 3 , sendo que as respostas " 0 " nunca ou "1" - às vezes, indicam um perfil negativo (não saudável) com risco à saúde, já as respostas "2" - quase sempre e “3” - sempre, indicam um perfil positivo (saudável), ou seja, com um melhor estilo ou hábito saudável. Desta forma o Pentáculo é colorido de acordo com o valor de cada resposta, zero indica que o participante não irá colorir o item no Pentáculo. Com a pontuação "1" é colorido do centro até o primeiro círculo; com a pontuação "2", do centro até o segundo círculo e com a pontuação "3”, do centro até o terceiro círculo. Quanto mais preenchido e colorido estiver o Pentáculo, melhor é considerado o PEVI do indivíduo (Nahas et al. 2000).

A qualidade de vida foi analisada pelo questionário SF-36 que avalia as condições da saúde em 8 domínios: 1) capacidade funcional em dez itens; 2) limitação por aspectos físicos em quatro itens; 3) dor em dois itens; 4) estado geral de saúde em cinco itens; 5) vitalidade em quatro itens; 6) aspectos sociais em dois itens; 7) limitação por aspectos emocionais em três itens e 8) saúde mental em cinco itens. Para avaliar os resultados, foi atribuído um escore para cada questão, objetivando transformá-lo numa escala graduada de 0 a 100, onde zero (0) corresponde a uma pior QV e cem (100) a uma melhor QV, possibilitando a análise individual de cada dimensão (Ciconelli et al.1 999).

A AF habitual foi avaliada pelo Questionário Internacional de Atividade Física (IPAQ) - versão curta em português, contendo perguntas referentes a prática cotidiana de $\mathrm{AF}$ na última semana em relação à frequência e duração de $\mathrm{AF}$ classificando como leve, moderadas e vigorosas e avalia o tempo da posição sentada ao longo e no final de uma semana. Obtém-se a seguinte classificação: a) Muito ativo: (Vigorosa $\geq 5$ dias por semana e $\geq 30$ minutos por sessão ou vigorosa $\geq 3$ dias por semana $\mathrm{e} \geq 20$ minutos por sessão, mais moderada e/ou caminhada $\geq 5$ dias por semana $\mathrm{e} \geq 30$ minutos por sessão); b) Ativo: (Vigorosa $\geq 3$ dias por semana e $\geq 20$ minutos por sessão, e/ou moderada ou caminhada $\geq 5$ dias por semana e $\geq 30$ 
minutos por sessão, e/ou qualquer atividade somada $\geq 5$ dias por semana e $\geq 150$ minutos por semana - caminhada, moderada e vigorosa); c) Irregularmente ativo A: (aquele que cumpriu apenas um dos critérios de recomendação quanto à frequência ou duração - 5 dias por semana ou 150 minutos por semana); d) Irregularmente ativo B: (aquele que não cumpriu nenhum dos critérios quanto à frequência ou duração) e e) Sedentário: (aquele que não realizou nenhuma atividade física por pelo menos 10 minutos contínuos durante a semana (Matsudo et al. 2002).

O projeto de pesquisa foi aprovado pelo Comitê de Ética da Universidade de Franca registrada sob o protocolo CAAE no. 65332517.4.0000.5495 e parecer no.581.894, obedecendo a resolução 466/12 do Conselho Nacional de Saúde.

\section{Análises estatísticas}

Inicialmente, os dados foram submetidos ao teste de normalidade Shapiro-Wilk. Os dados normais foram analisados por estatística paramétrica e os não normais com estatística não paramétrica. Os dados foram apresentados como média e desvio padrão e mediana e intervalo interquartil (25 e 75\%). As correlações entre o Pentáculo do bem-estar e o SF - 36 com a idade, massa corporal, estatura, IMC, tempo sentado na semana e no final de semana e IPAQ, foram realizados pelo teste Sperman. As associações entre os indicadores do IPAQ com a idade, IMC, carga horária semanal, tempo de serviço, Pentáculo do bem-estar e SF - 36, foram realizadas pelo teste Exato de Fisher, sendo avaliado a Odds Ratio (OR) com intervalo de confiança de (IC) de 95\%. As estatísticas foram realizadas pelo programa GraphPad Prism 6.0. O índice de significância adotado foi de $\mathrm{p}<0,05$.

\section{Resultados}

A amostra foi comporta por 93 docentes $\geq 40$ anos, a Tabela 1 apresenta as variáveis; idade, massa corporal, estatura e IMC. Também estão expostos a carga horária semanal e o tempo de serviço das professoras de ensino fundamental das escolas de Barretos, interior de São Paulo. Destaca-se que as professoras foram classificadas pelo IMC com sobrepeso e apresentam carga horária semanal acima de 40 horas semanais.

Tabela 1. Variáveis descritivas das participantes em relação a idade, massa corporal, estatura, IMC, carga horária semanal e tempo de serviço. $(n=93)$.

\begin{tabular}{lcc}
\hline Variáveis & Média \pm DP & Mediana (IQ 25 - 75\%) \\
\hline Idade (anos) & $43,5 \pm 7,8$ & $44(37-50)$ \\
MC (kg) & $70,6 \pm 12,7$ & $70(62-78)$ \\
Estatura (m) & $1,62 \pm 0,05$ & $1,63(1,58-1,65)$ \\
IMC & $26,9 \pm 4,8$ & $26,1(23,7-29,7)$ \\
CH/S (horas) & $40,4 \pm 13,4$ & $30(30-60)$ \\
TS (anos) & $16,5 \pm 7,9$ & $15(11-23)$ \\
\hline
\end{tabular}

MC, massa corporal; IMC, índice de massa corporal; CH/S, carga horária semanal; TS, tempo de serviço; DP, desvio padrão; IQ 25 - 75\%, intervalo interquartil de 25 e $75 \%$.

IMC $\leq 18,5$ ( baixo peso); IMC: 18,5 a 25,0 (peso adequado); IMC: 25,0 à 29,9 (sobrepeso); IMC: 30 à 34,9 (obesidade grau I); IMC: 35 à 39,9 (obesidade grau II); IMC: $\geq 40$ (obesidade grau III). Fonte: Autores.

Na Tabela 2 são apresentados os resultados do PEVI pelo Pentáculo do bem-estar, QV pelo SF - 36, e tempo de permanência sentado durante e aos finais de semana das professoras indicados pelo IPAQ. Em relação ao Pentáculo do bemestar, é observado que as professoras estão classificadas abaixo do nível considerado saudável. Sobre o SF - 36, foram consideradas próximas ao valor de referência determinado pelo questionário, que as classificam com boa qualidade de vida. 
Outra questão a ser observada é o tempo que as professoras ficarem sentadas, tanto durante a semana como nos finais de semana.

Tabela 2. Informações das participantes sobre o Pentáculo do bem-estar, SF - 36 e tempo sentado durante aos finais de acordo com o IPAQ versão curta. $(\mathrm{n}=93)$.

\begin{tabular}{|c|c|c|}
\hline Variáveis & Média \pm DP & Mediana (IQ 25-75\%) \\
\hline \multicolumn{3}{|l|}{ Pentáculo do bem-estar } \\
\hline Nutrição & $1,57 \pm 0,8$ & $2(1-2)$ \\
\hline Atividade Física & $0,94 \pm 0,9$ & $1(0-1)$ \\
\hline Comportamento Preventivo & $2,53 \pm 0,9$ & $3(2-3)$ \\
\hline Relacionamento Social & $2,05 \pm 0,9$ & $2(1-3)$ \\
\hline Controle do Estresse & $1,68 \pm 0,9$ & $2(1-2)$ \\
\hline Média geral das variáveis do Pentáculo & $1,75 \pm 0,5$ & $2(1-2)$ \\
\hline \multicolumn{3}{|l|}{ SF - 36} \\
\hline Capacidade Funcional & $80,2 \pm 17,1$ & $85(70-95)$ \\
\hline Limitação por aspectos físicos & $82,8 \pm 30,2$ & $100(75-100)$ \\
\hline Dor & $66,2 \pm 24,1$ & $72(43-84)$ \\
\hline Estado geral de saúde & $69,5 \pm 19,0$ & $72(57-82)$ \\
\hline Vitalidade & $57,4 \pm 21,5$ & $60(40-75)$ \\
\hline Aspectos Sociais & $74,5 \pm 25,3$ & $75(62-100)$ \\
\hline Limitação por aspectos emocionais & $77,8 \pm 36,6$ & $100(66-100)$ \\
\hline Saúde Mental & $67,0 \pm 20,0$ & $68(56-84)$ \\
\hline Média geral das variáveis do $S F$ - 36 & $71,9 \pm 6,5$ & $73(69-96)$ \\
\hline \multicolumn{3}{|l|}{ Tempo sentado segundo IPAQ } \\
\hline Durante a Semana (minutos) & $260,3 \pm 175,2$ & $240(120-300)$ \\
\hline Durante o final de semana (minutos) & $462,6 \pm 748,8$ & $300(240-480)$ \\
\hline
\end{tabular}

Legenda: SF - 36, 36-Item Short Form Survey; IPAQ, Questionário Internacional de Atividade Física; IQ 25 - 75\%, intervalo interquartil de 25 e $75 \%$; DP, desvio padrão. Fonte: Autores.

Em relação ao IPAQ, foi observado que a grande maioria das professoras $(73,2 \%)$ são classificadas como irregularmente ativas ou sedentárias (Tabela 3).

Tabela 3. Classificação do nível de atividade física das participantes segundo o IPAQ versão curta. (n=93).

\begin{tabular}{lcc}
\hline IPAQ & $\mathbf{N}$ & $\mathbf{\%}$ \\
\hline Muito ativa & 7 & 7,5 \\
Ativa & 18 & 19,3 \\
Irregularmente ativa A & 25 & 26,9 \\
Irregularmente ativa B & 22 & 23,7 \\
Sedentária & 21 & 22,6 \\
Total & $\mathbf{9 3}$ & $\mathbf{1 0 0 , 0}$ \\
\hline
\end{tabular}

Legenda: IPAQ: Questionário Internacional de Atividade Física; N, número. Fonte: Autores.

Na Tabela 4 são apresentados os dados da correlação do Pentáculo de bem-estar e do SF - 36 com variáveis antropométricas, tempo de trabalho na semana e de carreira, comportamento sedentário e IPAQ. Em nenhum dos testes foram observadas significâncias ( $\mathrm{p}>0,05)$. 
Tabela 4. Correlação do Pentáculo do bem-estar e do SF - 36 com as variáveis antropométricas, carga horária semanal, tempo de serviço em anos, tempo sentado na semana e no final de semana e em relação ao IPAQ. $(n=93)$.

\begin{tabular}{lcc}
\hline Variáveis & Pentáculo do bem-estar & SF -36 \\
\hline Idade (anos) & $\mathrm{r}=0,03(\mathrm{p}=0,70)$ & $\mathrm{r}=-0,15(\mathrm{p}=0,13)$ \\
MC (kg) & $\mathrm{r}=-0,02(\mathrm{p}=0,78)$ & $\mathrm{r}=-0,10(\mathrm{p}=0,31)$ \\
Estatura (m) & $\mathrm{r}=0,07(\mathrm{p}=0,47)$ & $\mathrm{r}=-0,05(\mathrm{p}=0,63)$ \\
IMC & $\mathrm{r}=-0,08(\mathrm{p}=0,39)$ & $\mathrm{r}=-0,08(\mathrm{p}=0,41)$ \\
CH/S (horas) & $\mathrm{r}=-0,19(\mathrm{p}=0,06)$ & $\mathrm{r}=-0,05(\mathrm{p}=0,60)$ \\
TS (anos) & $\mathrm{r}=0,07(\mathrm{p}=0,47)$ & $\mathrm{r}=-0,09(\mathrm{p}=0,38)$ \\
Sentado na Semana (min) & $\mathrm{r}=0,08(\mathrm{p}=0,44)$ & $\mathrm{r}=-0,06(\mathrm{p}=0,50)$ \\
Sentado no FDS (min) & $\mathrm{r}=0,11(\mathrm{p}=0,27)$ & $\mathrm{r}=-0,04(\mathrm{p}=0,66)$ \\
Ativas & $\mathrm{r}=-028(\mathrm{p}=0,16)$ & $\mathrm{r}=-0,10(\mathrm{p}=0,61)$ \\
Não ativas & $\mathrm{r}=-0,003(\mathrm{p}=0,97)$ & $\mathrm{r}=0,03(\mathrm{p}=0,80)$ \\
\hline
\end{tabular}

MC: massa corporal; IMC: índice de massa corporal; CH/S: carga horária semanal; TS: tempo de serviço; FDS: fim de semana; min: minutos; SF - 36: 36-Item Short Form Survey; Ativas: Muito Ativa + Ativa; Não ativas: Irregularmente Ativa + Sedentária. Fonte: Autores.

Na Tabela 5 é apresentado a associação entre o IPAQ com o tempo de serviço e com o Pentáculo do bem-estar. Foi observado que as professoras que apresentam mais que 11 anos de docência são mais ativas fisicamente. Em contrapartida, as professoras insuficientemente ativas apresentam escores no Pentáculo do bem-estar considerados prejudiciais para a saúde.

Tabela 5. Associação entre o IPAQ com a idade, IMC, carga horária semanal, tempo de serviço, Pentágono do bem-estar e SF $-36 .(n=93)$.

\begin{tabular}{|c|c|c|c|c|}
\hline Variáveis & $\begin{array}{l}\text { Ativas } \\
\text { n (\%) }\end{array}$ & $\begin{array}{c}\text { Não Ativas } \\
\text { n }(\%)\end{array}$ & OR (IC 95\%) & $\mathbf{p}$ \\
\hline \multicolumn{5}{|l|}{ Idade } \\
\hline$\leq 44$ anos & $11(44)$ & $42(61,7)$ & \multirow{2}{*}{$0,48(0,19-1,2)$} & \multirow{2}{*}{0,15} \\
\hline$\geq 45$ anos & $14(56)$ & $26(38,3)$ & & \\
\hline \multicolumn{5}{|l|}{ IMC } \\
\hline$\leq 24,9$ & $11(44)$ & $26(38,3)$ & \multirow{2}{*}{$1,26(0,50-3,21)$} & \multirow{2}{*}{0,63} \\
\hline$\geq 25,0$ & $14(56)$ & $42(61,7)$ & & \\
\hline \multicolumn{5}{|l|}{$\mathrm{CH} / \mathrm{S}$} \\
\hline$\leq 30$ horas & $14(56)$ & $40(58,8)$ & \multirow{2}{*}{$0,89(0,35-2,24)$} & \multirow{2}{*}{0,81} \\
\hline$\geq 31$ horas & $11(44)$ & $28(41,2)$ & & \\
\hline \multicolumn{5}{|l|}{ TS } \\
\hline$\leq 10$ anos & $1(4)$ & $19(27,9)$ & \multirow{2}{*}{$0,10(0,01-0,85)$} & \multirow{2}{*}{0,01} \\
\hline$\geq 11$ anos & $24(96)$ & $49(72,1)$ & & \\
\hline \multicolumn{5}{|l|}{ Pentáculo } \\
\hline$\leq 1$ & $13(52)$ & $51(75)$ & \multirow{2}{*}{$0,36(0,13-0,94)$} & \multirow{2}{*}{0,04} \\
\hline$\geq 2$ & $12(48)$ & $17(25)$ & & \\
\hline \multicolumn{5}{|l|}{ SF - 36} \\
\hline$\leq 69$ & $9(36)$ & $27(39,7)$ & \multirow{2}{*}{$0,85(0,33-2,21)$} & \multirow{2}{*}{0,81} \\
\hline$\geq 70$ & $16(64)$ & $41(60,3)$ & & \\
\hline
\end{tabular}

CH/S: carga horária semanal; TS: tempo de serviço; IMC: índice de massa corporal; SF - 36: 36-Item Short Form Survey. n: número; OR: Odds Ratio; IC 95\%: Intervalo de confiança de 95\%. Fonte: Autores.

\section{Discussão}

O presente estudo avaliou a influência do trabalho docente no ensino fundamental em indicadores de saúde de professoras do ensino fundamental.

Embora a QV tenha sido classificada como "boa", alguns indicadores importantes foram considerados abaixo da média, como dor, estado geral de saúde, vitalidade e saúde mental. 
Foi possível observar que estas professoras estão com sobrepeso, carga horária semanal acima do que sugere as leis trabalhistas, estão com PEVI considerado insatisfatório, em sua maioria ficam muito tempo sentadas e são consideradas insuficientemente ativas ou sedentárias.

Não foram observadas correlação entre todas as variáveis estudadas. Entretanto, foi identificado associação entre aumento de experiência docente com maiores níveis de AF, e inatividade com baixo nível de estilo ou hábitos nocivos à saúde.Os profissionais investigados no presente estudo apresentaram idade média de 43,5 \pm 7,8 anos, sendo ligeiramente superior à média de idade dos professores de educação básica nacional (INEP, 2017). Um importante fator encontrado nas variáveis da população investigada foi a prevalência de sobrepeso, sendo um indicativo que os professores estão à cima do peso desejável para manutenção da saúde e o que os predispõem a uma maior ocorrência de outras doenças crônicas não transmissíveis, como hipertensão e diabetes, nesta população (Canaan et al. 2010). Concordando com os nossos achados outros estudos semelhantes aplicados em outros munícipios brasileiros demonstraram que professores do ensino público mostraram prevalência de $58 \%$ de sobrepeso e $20 \%$ de obesidade no município de Viçosa-MG (Oliveira et al., 2011), sobrepeso/obesidade 47\% no munícipio Jequié-BA (Rocha et al.2 015) e 47\% no munícipio de Vitória da Conquista-BA (Ribeiro, 2018). O excesso de peso desta classe profissional parece ocorrer devido ao baixo nível de atividade física, presença de estresse e hábitos alimentares inadequados, como elevada ingestão de açucares e gordura (Moy et al. 2014, Vieira et al. 2020).

O perfil do estilo de vida individual foi avaliado através do Pentáculo do Bem-Estar (PBE) por meio do questionário que inclui características nutricionais, atividade física, comportamento preventivo e controle de stress e cada dimensão é avaliada através de três questões, a média das respostas através do questionário pode ser classifica 0 a 1 insatisfatório, 1 a 2 regular e 2 a 3 satisfatório (Nahas, 2006). Nossos resultados mostraram que a média do PBE apresentou o valor de 1,75 e isto ocorreu pelos valores baixos apresentados principalmente pela nutrição, atividade física e controle do estresse. Segundo (Coutinho, Foumel, Puntel, 2013) observaram que 111 professores do da rede pública estadual 86\% apresentaram estilo de vida negativo através (PBE) sendo que os fatores atividade física, nutrição e comportamento preventivo contribuíram para este resultado sendo identificado que a carga de trabalho teria um papel importante sobre o estilo de vida. Apesar da média da carga horária de trabalho dos profissionais investigados foi 40,4 horas, esta carga horária corresponde apenas a carga cumprida na escola. Estes profissionais na maioria das vezes, desenvolvem atividades em casa que a excede a carga horária prevista. Esta condição pode estar relacionada ao estilo de vida negativo encontrado no PBE.

O questionário SF-36 foi utilizado como ferramenta para avaliar a qualidade de vida dos professoras do ensino público (Karlsson, Sullivan, 2001). A média geral das variáveis do SF-36 foi de 71,9 mostrando que está ligeiramente superior a 70 que é valor limítrofe para classificação de uma boa qualidade de vida. Embora os valores do SF-36 sejam superiores a 70, algumas variáveis como dor, estado geral de saúde, vitalidade e saúde mental apresentaram resultados inferiores a 70. Segundo Moreira, Santino, Moraes (2017), professores do ensino fundamental do munícipio de Campina Grande mostraram scores baixos nas variáveis dor, estado geral da saúde e vitalidade. Possíveis fatores como desvalorização profissional associada ao baixo salário, carga horárias semanal acima ao vínculo, depressão e sintomatologia de dores musculo esquelética em decorrência da má postura influenciam a qualidade de vida destes profissionais (Ferreira-Costa \& Pedro-Silva, 2019; Berber et al., 2005; Pereira et al. 2014). Estudo recente aponta que novas pesquisa sobre a influência do trabalho na saúde mental deve ser realizadas, pois este tema, pois pode auxiliar na elaboração e implementações que que venham a contribuir para a promoção da saúde mental destes docentes (Lourenço et al., 2020).

A classificação do nível de atividade física foi avaliada através do questionário IPAQ, o qual, mostrou que 75\% das professoras eram sedentárias ou irregularmente ativas e, apenas $25 \%$ ativas ou muito ativas. Isto mostra que as professoras não atingem as recomendações de 150 minutos de atividade física por semana para promoção da saúde (ACMS, 2014). Da mesma forma, Brito et al. (2011) observaram baixo nível de atividade física de 1.681 professores da cidade de São Paulo. O mesmo 
resultado foi encontrado por Silva et al. (2015) em profissionais da rede pública do munícipio de Cacoal. A alta carga de trabalho, há presença de dores articulares e coluna e depressão podem serem fatores que predispõe para a prática insuficiente de atividade física justificados pelos professores entrevistados (Folle \& Farias, 2012). Isto vai ao encontro dos nossos achados que por meio do PEB apresentando valores inadequados do controle do estresse e SF-36 pelos aspectos negativos encontrado nas variáveis dor, estado geral da saúde e saúde mental. Desta maneira, parece que a carga horária de trabalho e fatores decorrentes do ambiente de trabalho, tais como: iluminação, ruídos, temperatura, ventilação e até mesmo relações interpessoais, desencadeando fatores desfavoráveis que influenciam negativamente o bem-estar e qualidade de vida promovendo a inatividade física. Além disso, nossos resultados demostraram que houve uma associação entre as professoras insuficiente ativas e baixo scores no Pentáculo do bem-estar, indicando que a inatividade física, bem como um baixo volume semanal de atividade física está associado a prejuízos à saúde.

Limitações do estudo

Dentre às limitações do estudo destaca-se a necessidade de cautela para extrapolação dos resultados para os docentes que atuam em outro nível (ensino médio e superior), uma vez que apenas docentes do ensino fundamental compuseram a amostra estudada. Outra limitação foi a utilização do peso e estatura autorreferidos, o que pode ter subestimado ou superestimado os valores do índice de massa corporal.

\section{Conclusão}

O presente estudo mostrou a prevalência de sobrepeso sendo um indicativo que as profissionais entrevistadas não apresentam o peso desejável para uma boa saúde, o que os predispõe para um maior risco de desenvolverem doenças crônicas não transmissíveis. Isto está associado a elevada carga horária, bem como a fatores do ambiente de trabalho como dor, estresse, saúde mental, que leva ao estilo de vida desfavorável e prejudica os indicadores de qualidade de vida e assim influencia diretamente o nível de atividade física dos entrevistados. Portanto, intervenções, no âmbito da educação básica, visando à redução da carga horária, bem como a criação de ambientes adequados a atividade laboral são estratégias que podem influenciar à promoção da prática de atividade física e assim, repercutir em melhorias na qualidade de vida dos docentes. Assim, sugere-se a realização de futuras investigações considerando a intervenção e acompanhamento longitudinal quanto a prática de atividades físicas, para melhor compreender os benefícios dessa prática na saúde desta população.

\section{Agradecimentos}

O presente trabalho foi realizado com apoio da Coordenação de Aperfeiçoamento de Pessoal de Nível Superior Brasil (CAPES) - Código de Financiamento 001.

\section{Referências}

American College Sports Medicine (2014). Diretrizes do ACMS para os testes de esforço e sua prescrição. Guanabara Koogan.

Berber, J. D. S. S., Kupek, E., \& Berber, S. C. (2005). Prevalência de depressão e sua relação com a qualidade de vida em pacientes com síndrome da fibromialgia. Revista Brasileira de Reumatologia, 45 (2), 47-54.

Brito, W. F., Santos, C. L. D., Marcolongo, A. D. A., Campos, M. D., Bocalini, D. S., Antonio, E. L., \& Serra, A. J. (2012). Nível de atividade física em professores da rede estadual de ensino. Revista de Saúde Pública, 46(1), 104-109.

Coutinho, R. X., Folmer, V., \& Puntel, R. L. (2011). Estilo de Vida de professores de escolas públicas de Uruguaiana. Anais do Salão Internacional de Ensino, Pesquisa e Extensão, 3(2).

da Silva, L. M. S., Pereira, F. D., Novello, T. P., \& da Silva Silveira, D. (2018). Relação entre a desvalorização profissional e o mal-estar docente. RELACultRevista Latino-Americana de Estudos em Cultura e Sociedade, 4. 
Research, Society and Development, v. 11, n. 1, e1511123919, 2022

(CC BY 4.0) | ISSN 2525-3409 | DOI: http://dx.doi.org/10.33448/rsd-v11i1.23919

de Oliveira, R. A. R., Júnior, R. J. M., Tavares, D. D. F., Moreira, O. C., Lima, L. M., dos Santos Amorim, P. R., \& Marins, J. C. B. (2015). Prevalência de obesidade e associação do índice de massa corporal com fatores de risco em professores da rede pública. Revista Brasileira de Cineantropometria e Desempenho Humano, 17(6), 742-752.

Delcor, N. S., Araújo, T. M., Reis, E. J., Porto, L. A., Carvalho, F. M., Silva, M. O., \& Andrade, J. M. D. (2004). Condições de trabalho e saúde dos professores da rede particular de ensino de Vitória da Conquista, Bahia, Brasil. Cadernos de Saúde Pública, 20 (1), $187-196$.

Felden Pereira, É., Stefani Teixeira, C., Pelegrini, A., Meyer, C., Andrade, R. D., \& Lopes, A. D. S. (2014). Estresse relacionado ao trabalho em professores de educação básica. Ciencia \& trabajo, 16(51), 206-210.

Ferreira-Costa, R. Q., \& Pedro-Silva, N. (2019). Níveis de ansiedade e depressão entre professores do Ensino Infantil e Fundamental. Pro-Posições, 30.

de Farias, G., \& Folle, A. (2012). Nível de qualidade de vida e de atividade física de professores de escolas públicas estaduais da cidade de Palhoça (SC). Revista Mackenzie de Educação Física e Esporte, 11(1), 11-21.

Gomes Moreira, Anne Samilly, Amorim Santino, Thayla, \& Ferreira Tomaz, Alecsandra. (2017). Qualidade de Vida de Professores do Ensino Fundamental de urna Escola da Rede Pública. Ciencia \& trabajo, 19(58), 20-25.

Lourenço, V. R., Valente, G. S. C., \& Correa, L. V. (2020). Influências do trabalho na saúde mental docente da escola pública do Rio de Janeiro. Research, Society and Development, 9(6), e50963250-e50963250.

Moy, F. M., Hoe, V. C. W., Hairi, N. N., Buckley, B., Wark, P. A., Koh, D., \& Bulgiba, A. M. (2014). Cohort study on clustering of lifestyle risk factors and understanding its association with stress on health and wellbeing among school teachers in Malaysia (CLUSTer)-a study protocol. BMC public health, 14(1), $1-9$

Nahas, M. A. F., \& Física, V. A. (2001). Saúde e qualidade de vida: conceitos e sugestões para um estilo de vida ativo. Midiograf.

Oliveira, R. A. R. D., Mota, R. J., Tavares, D. D. F., Moreira, O. C., Lima, L. M., Amorim, P. R. D. S., \& Marins, J. C. B. (2015). Prevalence of obesity and association of body mass index with risk factors in public school teachers. Revista Brasileira de Cineantropometria \& Desempenho Humano, $17(5), 742-752$.

Pereira A. S. et al. (2018). Metodologia da pesquisa científica. UFSM.

Reis, E. J. F. B. D., Carvalho, F. M., Araújo, T. M. D., Porto, L. A., \& Silvany Neto, A. M. (2005). Trabalho e distúrbios psíquicos em professores da rede municipal de Vitória da Conquista, Bahia, Brasil. Cadernos de Saúde Pública, 21(5), 1480-1490.

Rezende, F. A. C., Rosado, L. E. F. P. L., Franceschinni, S. D. C. C., Rosado, G. P., \& Ribeiro, R. D. C. L. (2010). The body mass index applicability in the body fat assessment. Revista Brasileira de Medicina do Esporte, 16(2), 90-94.

Ribeiro, V. S. M. (2018). Prevalência de sobrepeso e obesidade em professores da rede municipal da cidade de Vitória da Conquista-Bahia. Revista Brasileira de Saúde Funcional, 6(1), 31-31.

Rocha, S. V., Cardoso, J. P., Santos, C. A. D., Munaro, H. L. R., Vasconcelos, L. R. C., \& Petroski, E. L. (2015). Sobrepeso/obesidade em professores: prevalência e fatores associados. Revista Brasileira de Cineantropometria \& Desempenho Humano, 17(4), 450-459.

Secretaria Municipal da Educação. Educação (2021). Escolas municipais. http://www.barretos.sp.gov.br/secretaria-educacao

Silva, L. O., Baia, F., Romanholo, R. A., \& Soares, W. N. (2015). Nível de sedentarismo em professores do ensino regular da rede pública do município de Cacoal-RO. Revista Brasileira de Prescrição e Fisiologia do Exercício (RBPFEX), 9(52), 166-174.

Silveira, K. A., Enumo, S. R. F., Paula, K. M. P. D., \& Batista, E. P. (2014). Estresse e enfrentamento em professores: uma análise da literatura. Educação em Revista, 30(4), 15-36.

Taft, C., Karlsson, J., \& Sullivan, M. (2001). Do SF-36 summary component scores accurately summarize subscale scores? Quality of Life Research, 10(5), $395-404$.

Vieira, M. R. M., Magalhães, T. A. D., Silva, R. R. V., Vieira, M. M., Paula, A. M. B. D., Araújo, V. B., \& Haikal, D. S. A. (2020). Hipertensão Arterial e trabalho entre docentes da educação básica da rede pública de ensino. Ciência \& Saúde Coletiva, 25, 3047-3061. 